\title{
Première mesure belge de la prévalence du téléphone portable sans dispositif main libre au volant
}

\section{First Belgian survey of the prevalence of hand held mobile phone while driving}

\author{
François Riguelle - Mathieu Roynard - Freya Slootmans \\ C IFSTTAR et Éditions NecPlus 2015
}

\begin{abstract}
Résumé La distraction au volant et, en particulier, l'usage du téléphone portable sont de plus en plus considérés comme des problématiques importantes de sécurité routière. Il est nécessaire de mieux en évaluer l'ampleur afin de pouvoir prendre des contre-mesures efficaces. Dans cette optique, l'Institut Belge pour la sécurité routière a mené en 2013 la première mesure de la prévalence du téléphone sans dispositif main libre au volant au niveau belge. Nous avons mis en place une méthodologie rigoureuse et reproductible basée, d'une part, sur des observations sur 126 sites en bord de route, et, d'autre part, sur 6 journées d'observation depuis un véhicule en circulation sur l'autoroute. L'étude a permis de relever les comportements de 41315 conducteurs de voiture, camionnette, camion ou bus. Contrairement à plusieurs études internationales similaires, nous n'avons observé que les conducteurs de véhicules en mouvement. Outre l'usage du téléphone, nous avons observé d'autres caractéristiques des usagers ou de leur véhicule et testé leur impact sur la probabilité de conduire avec le téléphone au volant à l'aide d'un modèle logistique. Le type de véhicule et le type de route s'avèrent deux prédicteurs significatifs de l'usage du téléphone portable au volant. Globalement, les résultats de cette étude montrent que le téléphone portable sans dispositif main libre est un problème en Belgique et permettent d'identifier quelques pistes d'action.
\end{abstract}

François Riguelle $(\bowtie)$

Agence wallonne pour la Sécurité routière

Avenue Comte de Smet de Nayer 14, 5000 Namur, Belgique

e-mail : francois.riguelle@awsr.be

Mathieu Roynard $(\bowtie)$

Agence wallonne pour la Sécurité routière

Avenue Comte de Smet de Nayer 14, 5000 Namur, Belgique

e-mail : mathieu.roynard@awsr.be

Freya Slootmans $(\square)$

Institut Belge pour la Sécurité Routière

Chaussée de Haecht 1405, 1130 Bruxelles, Belgique

e-mail : freya.slootmans@ibsr.be
Mots clés téléphone portable · distraction au volant . mesure de comportement - observation · Belgique

Summary Distracted driving and, more specifically, the use of mobile phones, are increasingly seen as important issues for road safety. It is therefore needed to better evaluate its prevalence in order to be able to implement effective countermeasures. In this context, the Belgian Road Safety Institute has carried out in 2013 the first survey about handheld mobile phone use while driving at the Belgian level. We have implemented a rigorous and reproducible methodology based on, on one hand, a roadside survey on 126 locations, and, on the other hand, 6 days of observation from a moving car on highways. The survey allowed us to register the behaviour of 41315 car, lorry, truck or bus drivers. Unlike many similar international studies, we only focused on drivers of moving vehicles. Beside mobile phone use, we observed other characteristics of the drivers and of their vehicle and tested their impact on the risk of mobile phone use by using a logistic model. The vehicle type and the road type are found both significant predictors of mobile phone use while driving. Overall, the results of this study show that handheld mobile phone use while driving is a problem in Belgium and allow identifying some measures against it.

Keywords Mobile phone - Distracted driving - Behaviour measurement $\cdot$ Roadside survey $\cdot$ Belgium

\section{Introduction}

La distraction au volant est un problème de sécurité routière reconnu et qui, avec les nouvelles technologies, est de plus en plus d'actualité. Les études internationales estiment que de $5 \%$ à $25 \%$ des accidents de la route sont liés à cette problématique [1] [2] [3] [4].

La distraction au volant peut être définie comme un glissement de l'attention du conducteur vers une activité autre que les activités qui sont critiques pour pouvoir 
effectuer une tâche de conduite en sécurité [1] [5] [6] [7]. De toutes les sources de distraction possibles, le téléphone portable, et son évolution plus récente le smartphone (qui représente $75 \%$ des ventes d'appareils de téléphonie mobile en Belgique en 2014 [8]), est l'élément le plus décrié. En une quinzaine d'années, son utilisation est passée d'anecdotique à un comportement récurrent et répandu chez presque tous, et donc aussi parmi de nombreux conducteurs. L'évolution technologique des téléphones a permis d'en accroître les fonctionnalités. Ils ne servent plus seulement à téléphoner et envoyer des messages, mais permettent de se connecter à internet et démultiplient les possibilités d'utilisation : courriels, outils de géolocalisation, réseaux sociaux et une quantité importante d'applications. Les automobilistes ont donc de plus en plus de tentations à utiliser leur téléphone en conduisant.

Cependant, l'être humain n'est pas capable de mener plusieurs tâches simultanément en restant parfaitement efficace pour chacune d'entre elles. Selon la théorie des ressources multiples, on peut distinguer quatre dimensions dans les traitements nécessaires pour accomplir des tâches : le type de code manipulé, la modalité sensorielle impliquée, l'étape de traitement d'information et le mode de réponse [9]. Certaines combinaisons sont plus compatibles que d'autres au niveau de l'allocation des ressources. Toutefois, même si deux tâches présentent des combinaisons compatibles, cela ne signifie pas qu'il n'y a aucune dégradation des performances quand on les exécute simultanément. Ainsi, des recherches en neuropsychologie mettent en évidence que le cerveau ne peut pas consacrer toute son attention à la fois à des tâches visuelles et auditives, pourtant identifiées comme compatibles par la théorie des ressources multiples [10]. En faisant appel à des ressources cognitive et auditive (ainsi que physique en cas d'absence de dispositif main libre), l'usage du téléphone portable ne permet donc pas d'être concentré à $100 \%$ sur la conduite.

Les performances de conduite des personnes en train de téléphoner sont donc affectées [1] [11] [12]. Tenir une conversation téléphonique a un effet notable sur le temps de réaction à des événements inattendus (qui augmente de 20 à $40 \%$ ) et rend les freinages plus brusques [13]. Il n'y a par contre pas d'unanimité scientifique pour affirmer que les conversations téléphoniques impactent le contrôle de la trajectoire et les distances inter-véhiculaires [14]. Globalement, les altérations du comportement de conduite ne sont pas très différentes selon que le conducteur tienne une conversation avec un téléphone à l'oreille ou en utilisant un dispositif main libre. Téléphoner avec un dispositif main libre provoque autant de distraction cognitive que lorsqu'on tient son téléphone en main [15].

La manipulation du téléphone a aussi un impact sur les performances de conduite. Tant la consultation que la composition d'un message peut provoquer une augmentation du temps de réaction et de freinage, de plus grandes variations de la position latérale du véhicule et de la distance par rapport au véhicule précédent et faire manquer ou mal interpréter des événements ou des signaux routiers [12] [13] [16] [17] [18]. L'avènement des smartphones à écran tactile fait que les utilisateurs ne peuvent plus de baser sur des touches et accordent donc plus d'attention visuelle à leur téléphone et moins à la route.

L'évaluation de l'impact du téléphone au volant et des altérations de comportement qu'il provoque sur le risque d'accident varie beaucoup en fonction des études [17] [19] [20]. Toutefois, en considérant ces études dans leur ensemble, nous pouvons estimer qu'une personne en train de téléphoner au volant court approximativement de 3 à 4 fois plus de risques d'avoir un accident qu'une personne qui ne le fait pas [17] [20] [21] [22].

En conséquence, le code de la route belge, comme dans tous les pays de l'Union Européenne à l'exception de la Suède, interdit explicitement l'usage d'un téléphone portable au volant en le tenant en main (article 8.4). Ce comportement constitue une infraction de niveau 2 passible d'une amende minimale de $110 €$. Il n'existe par contre pas en Belgique d'interdiction de téléphoner avec un dispositif main libre, qu'on utilise une oreillette ou pas.

La dangerosité que représente l'utilisation du téléphone portable au volant justifie que l'on s'intéresse à la prévalence de ce comportement, pour pouvoir en suivre l'évolution et identifier des groupes à risque. De nombreuses études évaluant l'ampleur de la problématique du téléphone au volant reposent sur les comportements rapportés (via des questionnaires ou des interviews) et non sur des observations en conditions réelles [23] [24] [25]. Ainsi, la mesure d'attitudes de l'IBSR (Institut Belge pour la sécurité routière) a révélé que l'utilisation auto-rapportée du téléphone au volant parmi les automobilistes belges avait augmenté significativement entre 2009 et 2012. En 2012, $45 \%$ des répondants avouaient qu'il leur était arrivé au moins une fois dans l'année précédente de téléphoner au volant sans dispositif main libre, $34 \%$ d'envoyer un message et $50 \%$ d'en lire un [26]. Ce type d'étude ne permet toutefois pas d'avoir une idée précise de la prévalence de ce comportement, qui dépend non seulement de la fréquence du comportement mais aussi de sa durée. C'est pourquoi l'IBSR a décidé de mener en 2013 une première étude sur le téléphone au volant à l'aide d'observations en bord de route. Celle-ci a permis d'obtenir une mesure nationalement représentative de la prévalence instantanée de l'utilisation du téléphone portable sans dispositif main libre au volant et de pouvoir la comparer à la prévalence d'autres comportements dangereux étudiés précédemment comme la vitesse excessive et la conduite sous influence d'alcool. Bien 
que cette étude représente une première pour la Belgique, l'estimation de la prévalence du téléphone portable au volant par des observations de terrain a déjà été réalisée dans de nombreux pays depuis plusieurs années [27] [28] [29] [30] [31]. Bien qu'utilisant des méthodes proches, ces études varient sur la nature exacte des comportements observés, les conditions de circulation pendant lesquelles les observations sont effectuées et dans la taille des échantillons récoltés. En se basant sur ces expériences passées, nous avons voulu mettre en place une étude la plus représentative possible pour l'utilisation du téléphone portable sans kit main libre au volant en Belgique.

Dans la suite de cet article, nous détaillons la méthode employée pour effectuer les observations et analyser les données récoltées, décrivons les résultats obtenus et discutons des implications pour les acteurs de la sécurité routière.

\section{Méthodologie}

\section{Principe général}

La mesure de comportement a reposé sur une phase d'observation ayant eu lieu du 16 septembre au 8 octobre 2013. La fréquence à laquelle les conducteurs se rendaient coupables de l'usage du téléphone sans dispositif main libre a été observée par des enquêteurs placés en bord de route ou dans un véhicule en circulation (dans le cas des autoroutes). Les indicateurs obtenus permettent de définir la prévalence instantanée des comportements. Par exemple, un taux d'utilisation du téléphone de 3,3\% en heure de pointe indique que, si l'on prenait un arrêt sur image du trafic à un moment donné en heure de pointe, 3,3\% des conducteurs seraient en train d'utiliser leur téléphone portable au volant.

Nous avons décidé de n'observer que des véhicules en mouvement. Lorsque les véhicules à l'arrêt sont observés, la prévalence estimée de l'utilisation du téléphone au volant est généralement nettement supérieure à celle obtenue quand on ne considère que des véhicules en mouvement, comme illustré par deux études anglaises réalisées sur des véhicules arrêtés à des feux de circulation [32] [33]. Quand un véhicule est à l'arrêt à un feu ou pris dans les embouteillages, son conducteur est, en effet, bien plus susceptible d'utiliser son téléphone - notamment pour consulter un message reçu peu de temps auparavant que lorsqu'il roule. Toutefois, même si consulter son téléphone portable à l'arrêt dans la circulation est interdit et peut présenter un certain danger (pensons notamment au conducteur de poids lourd qui ne verrait pas un cycliste se placer dans son angle mort à un feu de circulation), le risque reste bien moindre que quand le comportement est réalisé par le conducteur d'un véhicule en mouvement. Nous avons donc voulu estimer la prévalence du téléphone au volant dans le cas où ce comportement est le plus problématique plutôt que d'obtenir des chiffres plus élevés mais ne représentant pas des situations où le danger est élevé.

\section{Échantillonnage et déroulement des observations hors autoroute}

L'enquête sur les axes hors autoroute a été menée sur un échantillon aléatoire stratifié de 126 sites d'observation. Les strates sont définies comme une combinaison d'une région, d'un type de route et d'une plage horaire (Tableau 1). Elles ont été mises en place afin de garantir des effectifs statistiquement suffisants dans chaque sous-groupe.

Il y a trois régions administratives en Belgique : la Région de Bruxelles-Capitale (RBC), la Flandre et la Wallonie. La région bruxelloise a été volontairement suréchantillonnée (36 sites sur 126) par rapport à sa taille relative $(10,4 \%$ de la population belge et $1,2 \%$ du réseau routier national) de sorte de pouvoir calculer des indicateurs fiables pour cette région. Les 90 sites restants ont été répartis équitablement entre la Flandre et la Wallonie.

La stratification des types de route a été réalisée en prenant pour hypothèse que la vitesse et la longueur du trajet influençaient le comportement en matière de téléphone au volant. Nous avons donc effectué des observations aussi bien en agglomération qu'en dehors, en tenant compte des différentes limitations de vitesse (30, 50,70 et $90 \mathrm{~km} / \mathrm{h}) .104$ sites se trouvaient sur des routes à une voie de circulation et 22 sur des routes à double voie.

Par ailleurs, nous avons veillé à mener l'étude aussi bien en semaine que le week-end, tout en distinguant les heures de pointe (définies sur les plages suivantes : de $7 \mathrm{~h}$ à $9 \mathrm{~h}$ et de $16 \mathrm{~h}$ à $18 \mathrm{~h}$ ) des heures creuses. Le même nombre de sessions d'observations a été réalisé durant ces trois périodes. Aucune observation de nuit n'a été effectuée et ce, d'une part, pour une raison de faisabilité (observations très difficiles dans l'obscurité) et, d'autre part, par manque de pertinence (la littérature scientifique ne renseigne pas que le problème du téléphone portable au volant est plus ou moins fréquent la nuit par rapport à la journée).

Cet ensemble de 3 régions et 4 types de route définit 11 strates (nous n'avons pas échantillonné de routes à $90 \mathrm{~km} / \mathrm{h}$ en $\mathrm{RBC}$ car cette région en contient très peu). Pour chacune de ces strates, nous avons sélectionné aléatoirement des sites à partir d'une base de données du réseau routier belge extraite d'OpenStreetMap. Étant donné que cette dernière ne contient pas, de façon uniforme et fiable, la limitation de vitesse des segments de route, la sélection des endroits s'est déroulée en deux phases. Premièrement, un point aléatoire sur le réseau routier était sélectionné à l'aide du logiciel QGIS. Nous avons ensuite recherché sur Google Earth un 
Tableau 1 Éléments constitutifs des strates utilisées pour l'échantillonnage et nombre de sites concernés

\begin{tabular}{lll}
\hline Région & \multicolumn{1}{c}{ Type de route } & \multicolumn{1}{c}{ Plage horaire } \\
\hline Bruxelles-Capitale (36) & $30 \mathrm{~km} / \mathrm{h}$ en agglomération (36) & Semaine en heure de pointe (42) \\
Flandre (45) & $50 \mathrm{~km} / \mathrm{h}$ en agglomération (42) & Semaine hors heure de pointe (42) \\
Wallonie (45) & $70 \mathrm{~km} / \mathrm{h}$ hors agglomération (31) & Journée de week-end (42) \\
& $90 \mathrm{~km} / \mathrm{h}$ hors agglomération (17) & \\
& & \\
\end{tabular}

site présentant la limitation de vitesse souhaitée le plus proche possible du point échantillonné. Chaque site s'est ensuite vu assigner aléatoirement une des 3 plages horaires.

La vérification sur Google Earth servait également à garantir que les endroits choisis permettaient de réaliser des mesures dans de bonnes conditions. Nous avons choisi des sites où nous pensions observer des véhicules en mouvement (hors intersection) et où les enquêteurs pouvaient travailler en toute sécurité (présence de trottoir ou de bas-côté).

La campagne d'observation proprement dite a été déléguée par l'IBSR à l'institut de sondage Ipsos. L'IBSR a organisé un briefing pour informer les enquêteurs d'Ipsos de l'objectif de l'étude et de la méthodologie souhaitée. Les enquêteurs ont travaillé en duo et collecté les données sur chaque site pendant une heure. Sur les lieux où le trafic n'était pas très dense, les enquêteurs observaient les véhicules à deux et se concertaient pour remplir le formulaire de récolte de données. En cas de trafic dense, un des deux enquêteurs se consacrait exclusivement au remplissage du formulaire pendant que l'autre lui dictait ce qu'il devait encoder.

Les enquêteurs disposaient d'un formulaire papier composé de propositions à entourer. Pour chaque véhicule, quatre informations étaient notées :

- le type de véhicule : 4 catégories possibles (voiture/camionnette/camion/bus ou car). Nous n'avons pas observé les usagers vulnérables ;

- le genre du conducteur;

- la présence de passager : 2 cas de figure ont été codés (conducteur seul ou avec passager(s) et ce quel que soit le nombre, l'âge, le genre ou la place dans le véhicule) ;

- l'utilisation du téléphone portable sans dispositif main libre : trois distinctions possibles (téléphone à l'oreille (supposément en train de téléphoner)/téléphone à la main/pas de téléphone). Les enquêteurs avaient la consigne de ne coder la présence du téléphone que s'ils étaient sûrs de l'avoir observé et de coder «pas de téléphone » en cas d'hésitation. Les résultats de cette étude constituent donc une estimation conservative du niveau d'utilisation du téléphone portable au volant, certains cas ayant sans doute échappé aux enquêteurs à cause de leur caractère furtif. D'autres comportements potentiellement distractifs ont été relevés mais ne font pas l'objet de cet article : la cigarette au volant, les objets en main (autre que téléphone et cigarette) et les manipulations du tableau de bord. Un rapport reprend les résultats concernant ces autres comportements [34].

Dans la mesure du possible, les enquêteurs ont observé tous les véhicules passant dans un sens de circulation sur le site de mesure. Lorsque le flux de circulation était trop important pour tout observer, ils avaient la consigne d'au moins encoder le type de chaque véhicule pour lequel le comportement $n$ 'avait pu être mesuré. Ce comptage des véhicules a permis de produire des indicateurs représentatifs pour l'ensemble du trafic des sites sélectionnés. En pratique, les enquêteurs ont pu réaliser des observations complètes pour une grande majorité des véhicules. Les informations sur la présence de distraction et le genre ne manquaient respectivement que dans $1,2 \%$ et $0,9 \%$ des cas.

Enfin, des informations sur les conditions climatiques au moment des observations (brouillard, vent, précipitations, soleil) ont été également relevées.

\section{Échantillonnage et déroulement des observations sur autoroute}

La procédure mise en place sur autoroute était différente par rapport aux autres types de route. L'observation depuis le bord des autoroutes n'est possible qu'à partir de certaines aires de repos mais la vitesse atteinte par la plupart des véhicules ne permet pas de déterminer si les conducteurs utilisent leur téléphone portable. Nous avons donc opté pour des observations effectuées depuis un véhicule en mouvement dans le trafic. Deux personnes de l'IBSR ont été impliquées : un conducteur et un observateur placé à l'arrière du véhicule. Les mesures se déroulaient en deux phases de 30 minutes successives. La première consistait à circuler à $90 \mathrm{~km} / \mathrm{h}$ pour observer le comportement 
des conducteurs des véhicules dépassant le véhicule des enquêteurs. Lors de la seconde, le véhicule roulait à $120 \mathrm{~km} / \mathrm{h}$ (limitation de vitesse en vigueur sur les autoroutes belges) pour mesurer les véhicules moins rapides sur la bande de droite. La feuille d'encodage était identique à celle utilisée sur les sites hors autoroute.

Le processus de récolte de données depuis un véhicule en mouvement ne permet toutefois pas d'avoir une estimation correcte de la quantité de trafic et de la répartition entre les différents types de véhicule. Pour obtenir cette information, nous avons effectué un arrêt sur une aire de repos sur chaque portion d'autoroute située entre deux échangeurs importants. Nous procédions alors à un comptage du trafic par catégorie de véhicule pendant 10 minutes. Nous observions également les comportements des conducteurs de poids lourd pendant une période de 15 minutes. Ceux-ci circulant en grande majorité sur la bande de droite et à $90 \mathrm{~km} / \mathrm{h}$, l'observation était donc plus aisée que depuis l'habitacle de la voiture en mouvement sur l'autoroute (où la différence de hauteur avec la cabine des camions posait des problèmes de visibilité pour les enquêteurs).

Il n'y a pas eu de procédure d'échantillonnage effectuée pour les sessions sur autoroute. En 6 jours d'observation (4 en semaine et 2 le week-end), nous sommes parvenus à couvrir pratiquement l'entièreté des 1763 kilomètres du réseau autoroutier du pays.

\section{Analyse}

L'encodage informatique des formulaires a été réalisé par Ipsos. L'IBSR était en charge du traitement et de l'analyse des données.

Les analyses statistiques ont été réalisées à l'aide du logiciel Stata. Nous avons calculé la prévalence du téléphone portable au volant en pondérant les données brutes pour tenir compte de la part respective réelle des différents types de véhicule, de la quantité de trafic par site de mesure, de la part relative des différentes plages horaires et de la proportion de chaque type de route dans chaque région (en termes de kilomètres de réseau). L'information étant manquante concernant la part respective des routes à 30 et $50 \mathrm{~km} / \mathrm{h}$ en zone urbaine et celle des routes à 70 et $90 \mathrm{~km} / \mathrm{h}$ hors agglomération, seuls 3 coefficients de pondération différents ont été utilisés en fonction du type de route : un pour les routes en agglomération, un pour les routes hors agglomération et un dernier pour les autoroutes. Compte tenu des résultats obtenus par type de route (prévalence similaire pour les routes à 30 et $50 \mathrm{~km} / \mathrm{h}$ et pour les routes à 70 et $90 \mathrm{~km} / \mathrm{h}$ ), nous pouvons en déduire que ces regroupements ont eu peu d'influence sur l'indicateur agrégé pour l'ensemble des routes.
Outre le calcul de la prévalence dans la population globale des conducteurs et dans toute une série de sous-groupes, nous avons également défini quels étaient les prédicteurs significatifs de l'usage du téléphone au volant à l'aide de régressions logistiques. Ce procédé permet de comparer la probabilité d'occurrence du téléphone au volant pour un conducteur appartenant à une catégorie d'une variable testée (par exemple la catégorie « conducteur de camionnette » de la variable «type de véhicule ») avec la probabilité d'un conducteur appartenant à une autre catégorie, désignée comme catégorie de référence (dans notre exemple les automobilistes). Nous avons réalisé deux analyses successives en utilisant d'abord comme variable dépendante la présence/absence du téléphone à l'oreille, et ensuite celle du téléphone en main. Nous n'avons pas étudié l'interaction entre les deux phénomènes étant donné qu'aucun conducteur observé n'a présenté en même temps les deux comportements (ce qui aurait nécessité deux téléphones).

Ces modèles logistiques ont été spécifiés manuellement, ce qui permet, mieux qu'une procédure automatique, de prendre en compte la théorie existante concernant les facteurs liés à l'utilisation du téléphone sans dispositif main libre au volant. Nous avons constaté une très faible variation des Odds Ratio (OR) des prédicteurs maintenus dans le modèle quand nous avons retiré les prédicteurs à l'effet non significatif, ce qui indique l'absence probable d'effet de confusion entre les variables enlevées du modèle et celles restantes. Au final, les prédicteurs maintenus dans les modèles présentent tous au moins une catégorie avec un Odds Ratio significatif au seuil de $95 \%$, indiquant un lien statistique fort avec l'utilisation du téléphone sans dispositif main libre au volant.

\section{Résultats}

Un total de 41315 conducteurs a été observé lors de cette mesure de comportement. La grande majorité d'entre eux étaient des automobilistes $(80,8 \%$ soit un effectif de 33 400). 9,6\% conduisaient des camionnettes (3 950) et $8,0 \%$ des poids lourds (3 300). Les sites d'observation n'ayant pas été ciblés en fonction des itinéraires des transports publics, relativement peu de conducteurs de bus ou car ont pu être observés (370 soit $0,9 \%$ ). Nous nous garderons donc d'analyser trop en détail les résultats de ce petit échantillon.

Parmi l'ensemble des conducteurs, la prévalence du téléphone à l'oreille est de $2,0 \%[1,7 \% ; 2,3 \%]$ (effectif de 939 conducteurs) et celle de la manipulation du téléphone de $1,2 \%[1,1 \% ; 1,4 \%]$ (effectif de 666). La prévalence totale du téléphone au volant sans dispositif main libre est donc 
de $3,2 \%[2,8 \% ; 3,6 \%]$ (puisque personne ne présente les deux comportements en même temps).

La prévalence du téléphone au volant sans dispositif main libre varie significativement en fonction de plusieurs variables prédictives comme le révèlent les modèles logistiques. Le tableau 2 reprend le modèle qui concerne le téléphone à l'oreille et le tableau 3 celui qui porte sur la manipulation du téléphone.

Le type de véhicule est un prédicteur hautement significatif du fait de tenir une conversation avec le téléphone à l'oreille au volant. Toutes choses égales par ailleurs, les conducteurs de camionnette ou de poids lourd (OR respectifs de $2,136^{* * *}$ et $2,134^{* * *}$ ) ont une probabilité de tenir leur téléphone à l'oreille deux fois plus élevée qu'un automobiliste. À l'inverse, les conducteurs de bus ou de car présentent une probabilité significativement plus faible que les automobilistes. En ce qui concerne la manipulation du téléphone portable, seuls les conducteurs de camionnettes $\left(\mathrm{OR}=1,628^{*}\right)$ se distinguent significativement des automobilistes. Nous pensons toutefois que le taux de manipulation du téléphone portable parmi les conducteurs de poids lourd pourrait être légèrement sous-estimé à cause des cabines hautes rendant l'observation plus difficile pour les enquêteurs.

En termes de prévalence (Fig. 1), 2,7 \% des automobilistes (effectif de 1 164) conduisaient en utilisant leur téléphone portable sans dispositif main libre contre $5,6 \%$ des conducteurs de camionnette (239) et 6,3\% des conducteurs de poids lourds (194). Les conducteurs de bus ou de car présentaient peu de comportements illégaux concernant le téléphone au volant.

Nous constatons également des variations significatives dans les comportements observés en fonction du type de route (Fig. 2). On observe clairement trois ensembles distincts : les routes d'agglomération, les routes hors agglomération et les autoroutes. Tant pour le téléphone à l'oreille qu'en main, rouler sur une route hors agglomération ou sur autoroute augmente significativement la probabilité de se livrer à un comportement illégal avec son téléphone portable par rapport aux personnes roulant en zone 30 en agglomération. Plus particulièrement, les autoroutes sont des lieux ou la probabilité de manipuler son téléphone portable est très élevée : 6 fois plus qu'en zone 30 $\left(\mathrm{OR}=6,223^{* * *}\right)$. Les autoroutes présentent une prévalence très élevée de manipulation du téléphone portable $(3,6 \%)$ et un total de $6,6 \%$ de conducteurs y utilise leur téléphone sans dispositif main libre, soit une personne sur 15.

En croisant les variables «type de véhicule » et «type de route », un sous-groupe se distingue particulièrement par sa propension à utiliser le téléphone portable sans dispositif main libre au volant. Il s'agit des conducteurs de camionnette sur autoroute avec un pourcentage impressionnant de
$14,7 \%$ [11,1\%; 19,4\%] d'entre eux utilisant un téléphone sans dispositif main libre. De même, les automobilistes utilisent également plus souvent leur téléphone portable sans dispositif main libre sur autoroute $(5,7 \%$ contre une moyenne de 2,7\% pour l'ensemble des types de routes). Seuls les poids lourds présentent un comportement plus sûr sur autoroute avec un taux d'utilisation du téléphone sans dispositif main libre de 5,5\% contre 6,3\% en moyenne.

De façon un peu contradictoire par rapport aux résultats en termes de type de route, la prévalence du téléphone portable sans dispositif main libre au volant est significativement plus élevée en Région de Bruxelles-Capitale $(5,6 \%[4,8 \% ; 6,8 \%])$ qu'en Flandre et en Wallonie (respectivement 3,4\% [2,8\%;4,1\%] et 2,9\% [2,5\%; $3,5 \%])$. Pourtant, la région bruxelloise est essentiellement urbaine, ce qui semble être un facteur limitant la probabilité de conduire avec le téléphone en main ou à l'oreille. Bruxelles fait donc exception à ce phénomène, peut-être à cause de la congestion du trafic routier dans la capitale : même si les conducteurs n'ont pas été observés lorsqu'ils étaient à l'arrêt, les fréquents ralentissements à Bruxelles seraient peut-être propices à débuter un appel téléphonique ou manipuler son appareil (message, application...), ce comportement entamé à l'arrêt se poursuivant lorsque le véhicule est à nouveau en mouvement et susceptible d'être observé.

Le genre du conducteur est prédictif de la probabilité de manipuler son téléphone mais pas de tenir son téléphone à l'oreille. Les femmes présentent en effet une probabilité moins grande $\left(\mathrm{OR}=0,627^{* *}\right)$ que les hommes de manipuler leur téléphone portable au volant. Dans le modèle concernant le téléphone à l'oreille, le genre n'est, en revanche, pas apparu comme significatif.

Pourtant, quand on regarde les résultats en fonction du genre pour tous les types de véhicules confondus (Fig. 3, à gauche), nous constatons que les hommes téléphonent plus avec le téléphone à l'oreille $(2,2 \%)$ et manipulent plus leur téléphone $(1,4 \%)$ que les femmes (avec respectivement $1,6 \%$ et $0,8 \%$ ). Mais ce résultat est un effet de la prévalence élevée de l'utilisation du téléphone portable sans dispositif main libre parmi les conducteurs de camionnette et de poids lourd. En effet, les femmes sont fortement sous-représentées parmi les conducteurs de camionnettes ( $8,6 \%$ de l'échantillon sont des femmes) et de camions (1,4\% de femmes). 98,8\% des femmes observées pendant l'étude étaient des automobilistes contre seulement 74,6\% des hommes. Le résultat des hommes est donc «dégradé » par les plus mauvais comportements exhibés par les conducteurs de camionnette et de poids lourd. Une analyse spécifique pour les automobilistes (Fig 3, à droite), montre que la prévalence du téléphone à l'oreille est similaire entre les hommes et les femmes ( $1,6 \%$ et $1,5 \%)$. Une différence 


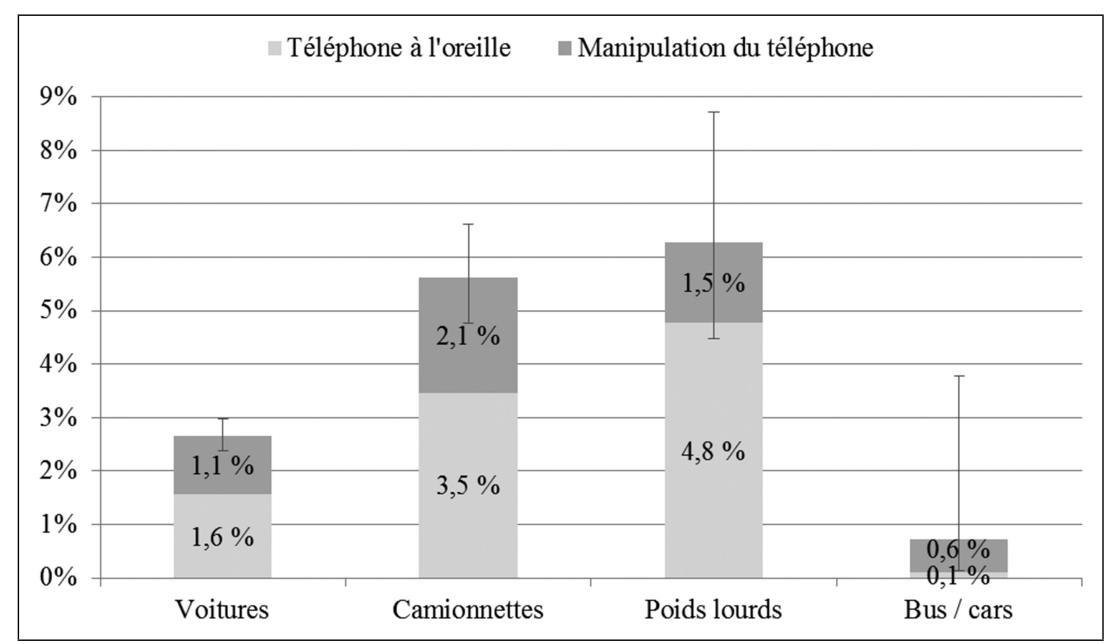

Fig. 1 Prévalence du téléphone portable sans dispositif main libre au volant en fonction du type de véhicule

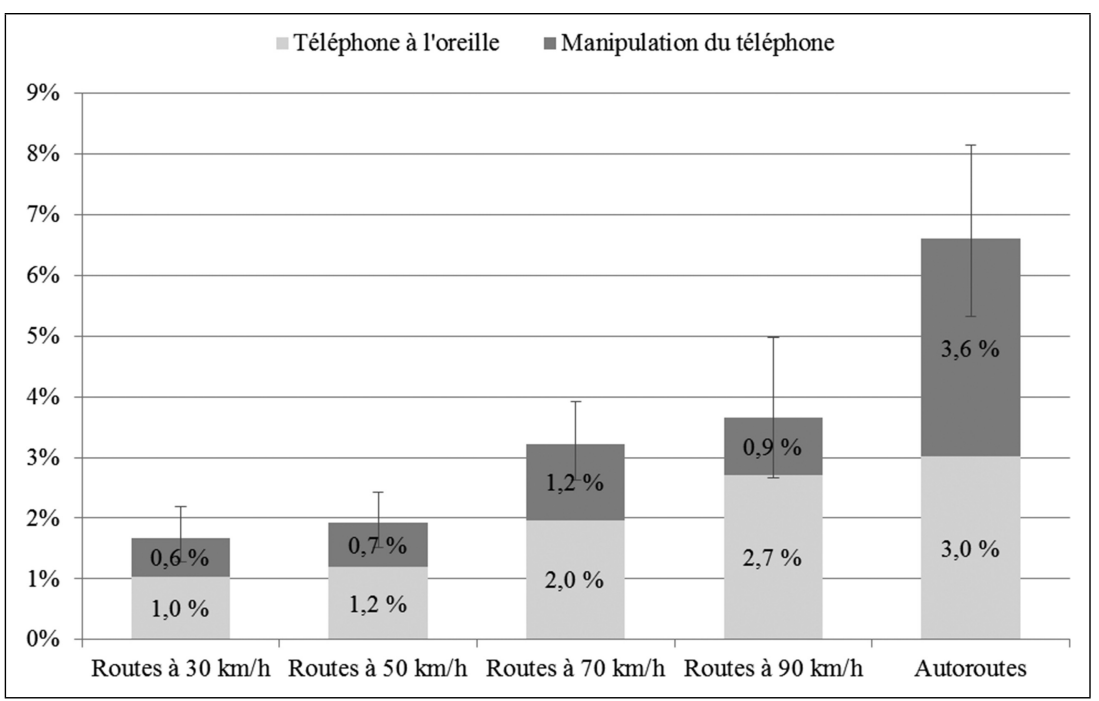

Fig. 2 Prévalence du téléphone portable sans dispositif main libre au volant en fonction du type de route

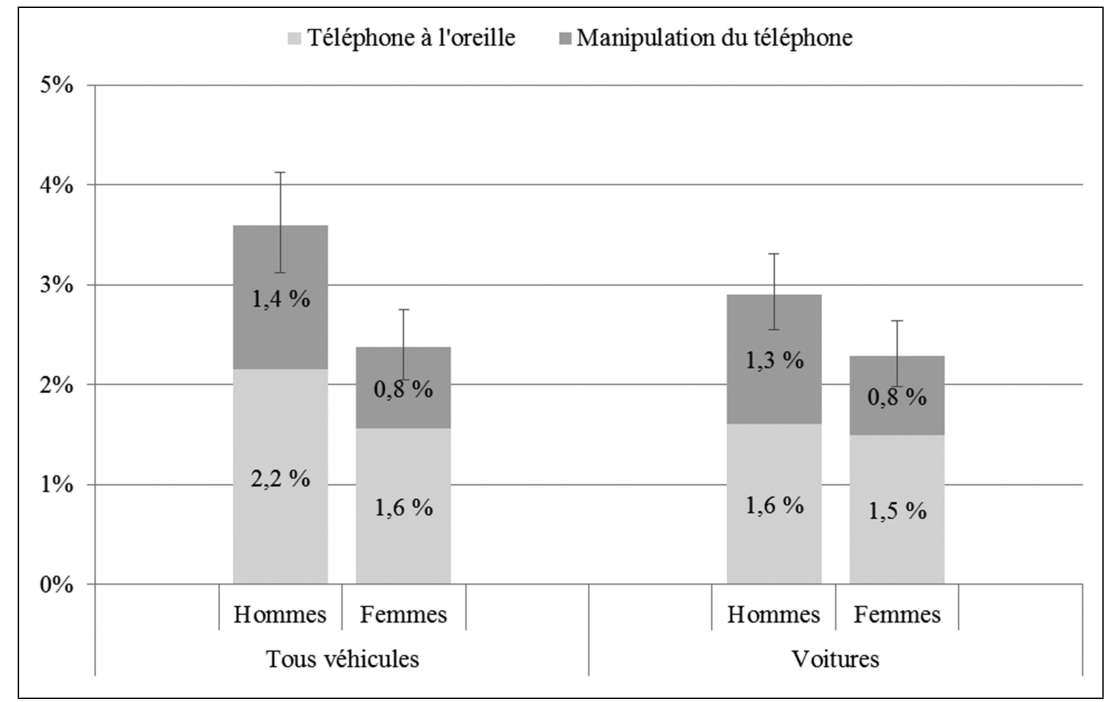

Fig. 3 Prévalence du téléphone portable sans dispositif main libre au volant en fonction du genre du conducteur 


\begin{tabular}{|c|c|c|c|c|}
\hline y : présence/absence du téléphone portable à l'oreille & Odds ratio & Erreur standard & $\mathbf{t}$ & $P>|t|$ \\
\hline \multicolumn{5}{|l|}{ Type de véhicule } \\
\hline \multicolumn{5}{|l|}{ Voiture - Catégorie de référence } \\
\hline Camionnette & $2,076 * * *$ & 0,23 & 6,7 & 0 \\
\hline Poids lourd & $2,134 * * *$ & 0,32 & 5,11 & 0 \\
\hline Bus/car & $0,106^{*}$ & 0,09 & $-2,59$ & 0,01 \\
\hline \multicolumn{5}{|l|}{ Présence de passager } \\
\hline \multicolumn{5}{|l|}{ Sans passager - Catégorie de référence } \\
\hline Avec passager(s) & $0,373 * * *$ & 0,05 & $-7,61$ & 0 \\
\hline \multicolumn{5}{|l|}{ Type de route } \\
\hline \multicolumn{5}{|l|}{ Agglo $30 \mathrm{~km} / \mathrm{h}$ - Catégorie de référence } \\
\hline Agglo $50 \mathrm{~km} / \mathrm{h}$ & 1,08 & 0,22 & 0,36 & 0,72 \\
\hline Hors agglo $70 \mathrm{~km} / \mathrm{h}$ & $1,787^{* *}$ & 0,37 & 2,84 & 0,01 \\
\hline Hors agglo $90 \mathrm{~km} / \mathrm{h}$ & $2,176^{* * *}$ & 0,5 & 3,41 & 0 \\
\hline Autoroutes $(120$ km/h) & $2,576^{* * *}$ & 0,59 & 4,16 & 0 \\
\hline \multicolumn{5}{|l|}{ Région } \\
\hline \multicolumn{5}{|l|}{$\mathrm{RBC}$ - Catégorie de référence } \\
\hline Flandre & $0,432 * * *$ & 0,07 & $-5,51$ & 0 \\
\hline Wallonie & $0,394 * * *$ & 0,06 & $-6,16$ & 0 \\
\hline Constante & $0,029 * * *$ & 0,01 & $-17,86$ & 0 \\
\hline$F(10,132)=30,37$ & Prob $>F=0,0000$ & & & \\
\hline
\end{tabular}

significative persiste en ce qui concerne la manipulation du téléphone (avec respectivement 1,3\% contre $0,8 \%$ ).

Sans surprise, transporter ou pas un passager modifie significativement le comportement en matière de téléphone au volant. Avoir au moins un passager à son bord diminue d'un facteur trois la probabilité de tenir son téléphone à l'oreille $(\mathrm{OR}=0,373 * * *)$ ou de manipuler son téléphone $(\mathrm{OR}=0,302 * * *)$. En moyenne, 4,1 \% [3,6 \% ; 4,6\%] des conducteurs voyageant seuls utilisent leur téléphone sans dispositif main libre au volant contre $1,3 \%[1,1 \% ; 1,6 \%]$ des conducteurs accompagnés. Ce phénomène peut trouver plusieurs explications. Le passager peut bien sûr prendre un appel ou écrire un message à la place du conducteur. Mais il y a sans doute également une forme de contrôle social qui entre en jeu, le conducteur ne voulant pas présenter un comportement dangereux devant son ou ses passagers. En outre, les conducteurs enclins à utiliser leur téléphone au volant pour «passer le temps » le feront moins s'ils ont quelqu'un avec eux pour discuter dans le véhicule.

Le moment d'observation a peu d'impact sur l'utilisation du téléphone sans dispositif main libre au volant. Celle-ci est certes moins fréquente pendant le week-end (prévalence de $2,0 \%$ [1,6\%;2,4\%]) qu'en semaine $(3,6 \%$ [3,2\% ; 4,1 \%]). Cependant, ces différences sont principalement dues à la composition différente du trafic entre la semaine et le week-end. En semaine, il y a en effet nettement plus de camionnettes et poids lourds et de personnes circulant sans passager que pendant le week-end. Or, ces éléments augmentent la probabilité d'utilisation du téléphone portable sans dispositif main libre au volant. Si l'on compare des catégories de conducteurs similaires (par exemple les automobilistes sans passager), les comportements en semaine et le week-end ne diffèrent pas fondamentalement.

Enfin, les variables liées aux conditions climatiques, également prises en compte dans l'analyse, ne s'avèrent pas être significatives. Notons toutefois que nous n'avons pas été confrontés à des conditions météorologiques extrêmes pendant l'étude (neige, verglas, grand vent), ce qui nous empêche d'affirmer que l'utilisation du téléphone portable sans dispositif main libre au volant est tout à fait indépendante des conditions climatiques. 


\begin{tabular}{|c|c|c|c|c|}
\hline y : présence/absence du téléphone portable en main & Odds ratio & Erreur standard & $\mathbf{t}$ & $\mathbf{P}>|\mathbf{t}|$ \\
\hline \multicolumn{5}{|l|}{ Type de véhicule } \\
\hline \multicolumn{5}{|l|}{ Voiture - Catégorie de référence } \\
\hline Camionnette & $1,628^{*}$ & 0,32 & 2,45 & 0,02 \\
\hline Poids lourd & 0,76 & 0,23 & $-0,91$ & 0,37 \\
\hline Bus/car & 0,84 & 0,78 & $-0,19$ & 0,85 \\
\hline \multicolumn{5}{|l|}{ Genre } \\
\hline \multicolumn{5}{|l|}{ Homme - Catégorie de référence } \\
\hline Femme & $0,627 * * *$ & 0,09 & $-3,29$ & 0 \\
\hline \multicolumn{5}{|l|}{ Présence de passager } \\
\hline \multicolumn{5}{|l|}{ Sans passager - Catégorie de référence } \\
\hline Avec passager(s) & $0,302 * * *$ & 0,06 & $-6,21$ & 0 \\
\hline \multicolumn{5}{|l|}{ Type de route } \\
\hline \multicolumn{5}{|l|}{ Agglo $30 \mathrm{~km} / \mathrm{h}$ - Catégorie de référence } \\
\hline Agglo $50 \mathrm{~km} / \mathrm{h}$ & 1,12 & 0,38 & 0,34 & 0,74 \\
\hline Hors agglo $70 \mathrm{~km} / \mathrm{h}$ & $2,074 * *$ & 0,53 & 2,88 & 0,01 \\
\hline Hors agglo $90 \mathrm{~km} / \mathrm{h}$ & 1,5 & 0,37 & 1,68 & 0,1 \\
\hline Autoroutes $(120$ km/h) & $6,223 * * *$ & 1,51 & 7,56 & 0 \\
\hline \multicolumn{5}{|l|}{ Région } \\
\hline \multicolumn{5}{|l|}{$\mathrm{RBC}$ - Catégorie de référence } \\
\hline Flandre & $0,294 * * *$ & 0,06 & $-6,1$ & 0 \\
\hline Wallonie & $0,297 * * *$ & 0,08 & $-4,59$ & 0 \\
\hline Constante & $0,028 * * *$ & 0,01 & $-12,91$ & 0 \\
\hline$F(10,132)=30,37$ & Prob $>F=0,0000$ & & & \\
\hline
\end{tabular}

\section{Discussion et conclusion}

La mesure de comportement a révélé que la prévalence de l'utilisation du téléphone portable sans dispositif main libre au volant était élevée : 3,2\% des conducteurs circulent tout en tenant un téléphone portable $(2,0 \%$ à l'oreille et $1,2 \%$ en manipulation). Il est difficile de déterminer si cette prévalence doit être considérée comme élevée en comparaison avec d'autres études internationales car peu d'études récentes ont utilisé une méthodologie similaire à celle de l'étude présente. Deux études comparables ont toutefois relevé des taux d'utilisation du téléphone sans dispositif main libre inférieurs à ceux de cette étude. En France, l'ONISR, qui réalise chaque année une mesure d'observation concernant le téléphone au volant, mesurait en 2012 une prévalence de $1,5 \%$ du téléphone tenu à l'oreille et de $0,5 \%$ du téléphone en manipulation [35]. De même, une étude anglaise rapportait une prévalence totale de l'utilisation du téléphone portable sans dispositif main libre au volant de $1,4 \%$ [36].

Comme signalé en introduction, le risque d'accident est multiplié entre 3 et 4 fois lorsque l'on téléphone au volant. Les résultats obtenus sont donc assez préoccupants pour la sécurité routière, surtout en se rappelant, qu'aux utilisations mesurées au cours de cette étude, se rajoute l'utilisation du téléphone avec dispositif main libre qui - bien que légale augmente également le risque d'accident.

Le téléphone sans dispositif main libre au volant est une infraction qui concerne beaucoup de conducteurs. Lors de la mesure d'attitude 2012 de l'IBSR, $45 \%$ des automobilistes avouaient qu'il leur arrivait de téléphoner au volant sans dispositif main libre, $34 \%$ qu'il leur arrivait de rédiger un message et $50 \%$ qu'il leur arrivait de lire un message [26]. Bien que téléphoner et envoyer des messages au volant sont des pratiques généralement considérées comme dangereuses par les usagers de la route, cela ne freine donc pas certains 
conducteurs à les adopter. La même étude évaluait aussi la norme sociale ressentie par les conducteurs. En regardant les résultats par région, on observe un lien entre la prévalence $\mathrm{du}$ téléphone portable sans dispositif main libre et la norme ressentie. À Bruxelles, où le téléphone portable au volant est le plus souvent observé, un pourcentage élevé de conducteurs $(82 \%)$ considère que téléphoner au volant sans dispositif main libre fait partie de la norme sociale, contre seulement $72 \%$ des Flamands et $71 \%$ des Wallons [26].

Un résultat marquant de cette étude est la prévalence élevée du téléphone portable sans dispositif main libre au volant parmi les conducteurs de camionnette ou de poids lourd. Ce résultat n'est pas une surprise car également rapporté par plusieurs autres études internationales [35] [36] [37] [38]. La plupart des conducteurs de camionnette et de camion se déplacent dans le cadre de leur travail, souvent en tant que conducteurs professionnels. Il y a donc certainement des actions à mener au niveau des entreprises et des indépendants pour les sensibiliser au danger et réduire l'utilisation du téléphone au volant. Par ailleurs, les employeurs ne doivent pas non plus exiger de leurs employés qu'ils soient joignables à tout moment, même sur la route.

Certaines études indiquent que tenir une conversation téléphonique au volant n'augmente heureusement pas autant le risque d'accident parmi les conducteurs professionnels que parmi les automobilistes [39] [40]. Téléphoner au volant est notamment utilisé par certains conducteurs professionnels comme une stratégie anti-somnolence. Envoyer des messages reste, en revanche, un comportement très risqué.

Il faut noter que plus de $40 \%$ des kilomètres parcourus par des poids lourds en Belgique le sont par des véhicules immatriculés à l'étranger. Beaucoup de ces camions étrangers ne font que traverser la Belgique en circulant exclusivement sur les autoroutes. Ces conducteurs étrangers présentent peut-être des comportements différents de ceux des Belges, ce qui pourrait influencer les résultats de l'étude, et plus particulièrement la mesure de prévalence du téléphone sans dispositif main libre sur autoroute.

Un autre résultat notable est la relation entre la prévalence du téléphone portable sans dispositif main libre au volant et le type de route. Celle-ci augmente avec la limitation de vitesse pour atteindre une valeur maximale de 6,6\% sur autoroute. Cela pourrait sembler étonnant car être distrait de sa tâche de conduite à haute vitesse est a priori plus dangereux qu'à plus basse vitesse. Mais il faut aussi tenir compte que les déplacements sur les routes à 30 et $50 \mathrm{~km} / \mathrm{h}$ se font en contexte urbain, où les conducteurs sont soumis à plus d'interactions avec les autres usagers de la route, ont ainsi plus de tâches à accomplir et sont plus susceptibles de rencontrer un événement inattendu. $\mathrm{Au}$ contraire, les routes hors agglomération et les autoroutes présentent plus souvent des sections où le conducteur a peu de tâches à accomplir, mis à part garder son attention sur la route et maintenir sa trajectoire. Par ailleurs, les conducteurs circulant sur des routes hors agglomération effectuent vraisemblablement de plus longs déplacements que les conducteurs en milieu urbain et sont donc moins enclins à reporter une communication téléphonique ou la consultation d'un message à la fin de leur trajet. Il leur est, également, moins facile de s'arrêter pour consulter leur téléphone portable.

$\mathrm{Au}$ niveau démographique, l'étude a mis en évidence une propension plus élevée des hommes que des femmes à manipuler leur téléphone portable au volant. Ce résultat n'est pas partagé par beaucoup d'études internationales. Au contraire, plusieurs études identifient une prévalence plus élevée du téléphone portable au volant parmi les femmes que les hommes [35] [38] [41]. Un autre aspect démographique pour lequel la littérature scientifique est beaucoup plus unanime est l'âge. Les études ayant étudié le lien entre l'âge et la prévalence du téléphone portable au volant mettent presque toujours en évidence que les jeunes conducteurs utilisent plus leur téléphone au volant que les conducteurs plus âgés [37] [38] [41] [42]. En Belgique, la mesure d'attitudes 2012 de l'IBSR indiquait que, plus les répondants étaient jeunes, plus ils déclaraient utiliser fréquemment leur téléphone au volant [26]. Cela valait tant pour le fait de téléphoner sans dispositif main libre que pour l'envoi et la lecture de messages. Néanmoins, au niveau des observations, il est extrêmement difficile d'évaluer l'âge des conducteurs. Cette variable n'a donc pas pu être incluse au niveau de cette mesure de comportement.

L'usage du téléphone au volant est un comportement susceptible de s'intensifier à l'avenir si des mesures concrètes ne sont pas prises. En outre, les évolutions technologiques actuelles tendent plutôt vers l'accroissement des sources de distraction au volant, via des véhicules hyperconnectés, véritables pôles multimédias. La plupart des nouvelles applications peuvent être activées vocalement, sans devoir quitter les mains du volant. Elles sont donc souvent présentées, à tort, comme inoffensives. De plus, le phénomène de distraction cognitive, pourtant très important, est souvent minimisé ou passé sous silence par les concepteurs. Cependant, la technologie peut aussi aider à lutter contre la distraction due au téléphone au volant. Des applications smartphone permettant de bloquer certaines fonctionnalités des téléphones, de filtrer les notifications des applications ou encore d'avertir les correspondants que le conducteur n'est pas en mesure de répondre à un appel commencent à se développer. Ces initiatives doivent être soutenues et les automobilistes encouragés à les utiliser.

Beaucoup de personnes ne conçoivent plus de ne pas être joignables tout le temps, en toutes circonstances, même au volant. Différentes études relèvent qu'il y a beaucoup 
de travail de communication nécessaire pour changer les attitudes et les normes sociales concernant le téléphone au volant [17] [43]. Les campagnes de prévention doivent donc redoubler d'effort pour faire comprendre et accepter aux conducteurs l'importance d'être concentré à $100 \%$ sur leur tâche de conduite. La sensibilisation doit particulièrement cibler les jeunes, très amateurs de nouvelles technologies et conduisant plus souvent avec le téléphone au volant, d'après les recherches nationales et internationales. Nous préconisons également de promouvoir, dès l'apprentissage à la conduite, une utilisation raisonnée des technologies lors de la conduite. La sensibilisation seule n'est toutefois pas suffisante pour minimiser les mauvais comportements en matière de téléphone au volant. Elle doit être accompagnée de solutions technologiques et d'une politique de sanction visant à accroître le risque objectif et subjectif d'être contrôlé [44].

Cette mesure de comportement sur l'utilisation du téléphone sans dispositif main libre au volant représentait une première en Belgique. Devant la multiplication des nouvelles technologies embarquées dans les véhicules, l'IBSR est appelé dans le futur à étendre les recherches sur les distractions en prenant en compte d'autres facteurs tels que les téléphones avec dispositifs main libre, les tablettes et tous les dispositifs multimédias présents dans les véhicules. Certains comportements liés ne sont pas mesurables avec une méthode classique d'observation le long des routes. Il faudrait donc étendre la gamme de questions posées lors des enquêtes d'attitudes et développer de nouvelles méthodologies de recherche, comme les études naturalistic driving ou en simulateur. L'IBSR a d'ailleurs l'intention d'étudier prochainement l'influence de différentes distractions sur la conduite sur base d'études en simulateur. Enfin, il serait intéressant d'effectuer une mesure de comportement concernant les sources de distraction auprès des usagers vulnérables.

\section{Références}

1. OMS (2011) L'utilisation des téléphones mobiles : la distraction au volant, un problème qui s'aggrave. Organisation Mondiale de la Santé, Genève, $48 \mathrm{p}$.

2. DaCoTA (2012) Driver distraction. Deliverable $4.8 \mathrm{f}$ of the EC FP7 project DaCoTA.

3. SWOV (2013) SWOV-Factsheet. Afleiding in het verkeer. Stichting Wetenschappelijk Onderzoek Verkeersveiligheid, Leidschendam, $6 \mathrm{p}$.

4. Meesmann U, Opdenakker E (2013) Comportements détournant l'attention chez les conducteurs professionnels. Institut Belge pour la Sécurité Routière - Centre de connaissance Sécurité Routière, Bruxelles, $45 \mathrm{p}$.

5. Ranney TA (2008) Driver Distraction: A Review of the Current State-of-Knowledge. National Highway Traffic Safety Administration, Washington DC, $25 \mathrm{p}$.
6. Stelling A, Hagenzieker MP (2012) Afleiding in het verkeer. Een overzicht van de literatuur. Stichting Wetenschappelijk Onderzoek Verkeersveiligheid, Leidschendam, $87 \mathrm{p}$.

7. Regan MA, Hallet C, Gordon CP (2011). Driver distraction and driver inattention: Definition, relationship and taxonomy. Accident Analysis and Prevention 43: 1771-1781.

8. GfK (2015) Communiqué de presse : 3 trends in the Belgian smartphone market. Consulté le 21 août 2015, à l'adresse http://www.gfk.com/be/news-and-events/press-room/pressreleases/Pages/3-trends-in-the-Belgian-smartphone-market.aspx.

9. Wickens CD (2002). Multiple resources and performance prediction. Theoretical Issues in Ergonomic Science 3(2): 159-177.

10. Shomstein S, Yantis S (2004). Control of attention shifts between vision and audition in human cortex. Journal of Neuroscience 24(47): 10702-10706.

11. Breen J. (2009) Car telephone use and road safety: final report. An overview prepared for the European Commission. Jeanne Breen Consulting, Bruxelles, $21 \mathrm{p}$.

12. Drews FA, Yazdani H, Godfrey CN et al. (2009) Text messaging during simulated driving. The Journal of Human Factors and Ergonomics 51: 762-770.

13. SWOV (2012) SWOV-Factsheet. Mobiel telefoongebruik door bestuurders. Stichting Wetenschappelijk Onderzoek Verkeersveiligheid, Leidschendam, $6 \mathrm{p}$.

14. Caird JK, Willness CR, Steel P, Scialfa C (2008) A meta-analysis of the effects of cell phones on driver performance. Accident Analysis and Prevention 40: 1282-1293.

15. Strayer DL, Watson JM, Drews FA (2011) Cognitive distraction while multi-tasking in the automobile. Psychology of Learning and Motivation 54: 29-58.

16. Reed N, Robbins R (2008) The effect of text messaging on driver behaviour: a simulator study. Transport Research Labatory, Crowthorne, $54 \mathrm{p}$.

17. Inserm (2011) Téléphone et sécurité routière. Expertise collective. Institut national de la santé et de la recherche médicale, Paris, $269 \mathrm{p}$.

18. Hagenzieker MP, Stelling A (2013) Schatting aantal verkeersdoden door afleiding. Stichting Wetenschappelijk Onderzoek Verkeersveiligheid, Leidschendam, $16 \mathrm{p}$.

19. Klauer SG, Dingus DR, Neale TA et al. (2006) The impact of driver inattention on near-crash/crash risk: An analysis using the 100-car naturalistic study data. National Highway Traffic Safety Administration, Washington DC, $226 \mathrm{p}$.

20. Elvik R (2011) Effects of Mobile Phone Use on Accident Risk: Problems of Meta-Analysis When Studies Are Few and Bad. Transportation Research Record 2236: 20-26.

21. Redelmeier DA, Tibshirani RJ (1997) Association between cellular telephone calls and motor vehicle collisions. The New England Journal of Medicine 336: 453-458.

22. McEvoy SP, Stevenson MR, McCartt AT et al. (2005) Role of mobile phones in motor vehicle crashes resulting in hospital attendance: A case-crossover study. British Medical Journal 331: 428-430.

23. Schroeder P, Meyers M, Kostyniuk L (2013). National survey on distracted driving attitudes and behaviors-2012. Rapport No. DOT HS 811 729. National Highway Traffic Safety Administration, Washington, DC.

24. Thulin H, Gustafsson S. (2004). Mobile phone use while driving. VTI rapport No. 490A. VTI, Linköping, Suède, $38 \mathrm{p}$.

25. Beirness DJ (2005). Distracted driving: The role of survey research. International Conference on Distracted Driving, Toronto.

26. Meesmann U, Boets S (2014) Fatigue et distraction due à l'usage $d u$ GSM. Résultats de la mesure d'attitudes en matière de sécurité routière menée tous les trois ans par l'IBSR. Institut Belge pour la Sécurité Routière - Centre de connaissance Sécurité routière, Bruxelles, $50 \mathrm{p}$. 
27. Horberry T, Bubnich C, Hartley L, Lamble D (2001). Drivers' use of hand-held mobile phones in Western Australia. Transportation Research Part F : Traffic Psychology and Behaviour 4 : 213-218.

28. Utter D (2001). Passenger vehicle driver cell phone use: Results from the fall 2000 national occupant protection use survey. Rapport no DOT HS-809-293. NHTSA, Washington, DC.

29. Bedford D, O'Farrell A, Downey J, McKown F (2005) The use of hand held mobile phones by drivers. Irish Med J: 98-248.

30. Hill JP (2005) A survey of mobile phone used by drivers. TRL Report $\mathrm{N}^{\circ}$ TRL635. TRR, Londres.

31. Observatoire national interministériel de la sécurité routière (2008) La sécurité routière en France : bilan de l'année 2007. ONISR, Paris.

32. Narine S, Walter LK, Charman SC (2010) Mobile phone and seat belt usage rates in London 2009. Published project report 418. Transport Research Laboratory, Wokingham, Royaume-Uni.

33. Scoons J (2012) Mobile phone and seat belt usage rates in Surrey 2012. Published project report PPR642. Transport Research Laboratory, Wokingham, Royaume-Uni.

34. Riguelle F, Roynard M (2014) Conduire sans les mains. Utilisation du GSM et d'autres objets pendant la conduite sur le réseau routier belge. Institut Belge pour la Sécurité Routière Centre de connaissance Sécurité Routière, Bruxelles, 51 p.

35. Observatoire national interministériel de la sécurité routière (2014) L'évolution du comportement des conducteurs : 2012 et années précédentes. Note de synthèse. ONISR, Paris, France, 9 p.

36. Walter L (2010) Seatbelt and mobile phone usage surveys: England and Scotland 2009. Department for Transport, Londres, Royaume-Uni, 49 p.
37. Narine S, Walter LK, Charman SC (2010) Mobile phone and seat belt usage rates in London 2009. Published project report 418. Transport Research Laboratory, Wokingham, Royaume-Uni, $44 \mathrm{p}$.

38. NHTSA (2015). Driver electronic device use in 2013. Rapport DOT HS 812 114. National Highway Traffic Safety Administration, Washington, DC, États-Unis, 8 p.

39. Olson R, Hanowski R, Hickman J, Bocanegra J (2009) Driver distraction in commercial vehicle operations. US Department of Transportation, Federal Motor Carrier Safety Administration, Washington DC, $285 \mathrm{p}$.

40. Hickman JS, Hanowksi RJ, Bocanegra J (2010) Distraction in Commercial Trucks and Buses: Assessing Prevalence and Risk in Conjunction with Crashes and Near-Crashes. US Department of Transportation, Federal Motor Carrier Safety Administration, Washington DC, $79 \mathrm{p}$.

41. McCartt AT, Hellinga LA, Strouse LM, Farmer CM (2010) Longterm effects of handheld cell phone laws on driver handheld cell phone use. Traffic Injury Prevention 11: 133-141.

42. Scoons J (2012) Mobile phone and seat belt usage rates in Surrey 2012. Published project report PPR642. Transport Research Laboratory; Wokingham, Royaume-Uni, $28 \mathrm{p}$.

43. Robertson R (2011) Distracted driving: so what's the big picture? Traffic Injury Research Foundation, Ottawa, Canada, 15 p.

44. Rowden P, Watson B (2013) Mobile Phone Use and Driving: The Message Is Just Not Getting Through. Actes de la conference "Australasian College of Road Safety Conference", Adelaide, Australie. 